\title{
Simulation Analysis and Experimental Research on Mechanical Properties of Water-Hydraulic Ball Valve
}

\author{
Yong Yang \\ Dalian Maritime University \\ Zengmeng Zhang (D zzm.zju@163.com) \\ Dalian Maritime University \\ Yinglong Chen \\ Dalian Maritime University \\ Yongjun Gong \\ Dalian Maritime University
}

\section{Research Article}

Keywords: Water-Hydraulic, Ball valve, Mechanical properties, CFD

Posted Date: January 19th, 2022

DOI: https://doi.org/10.21203/rs.3.rs-1207818/v1

License: (c) (1) This work is licensed under a Creative Commons Attribution 4.0 International License.

Read Full License 


\section{Title page}

\section{Simulation Analysis and Experimental Research on Mechanical Properties of Water-Hydraulic Ball Valve}

Yong Yang, received the B.Eng. degrees in mechanism design, manufacturing and automatization from Dalian Maritime University, Dalian, China, in 2018. Since 2018, he has been a master student with the Naval Architecture and Ocean Engineering College, Dalian Maritime University. His research interests include fluid power transmission and control, water hydraulics and control valves. E-mail: dlmu_yy@163.com

Zeng-Meng Zhang, received the Ph.D. degrees in mechatronic control engineering from Zhejiang University, Zhejiang, China, in 2009. He is currently an Professor with the Naval Architecture and Ocean Engineering College, Dalian Maritime University. His research interests include fluid power transmission and control, water hydraulics, artificial muscles, control valves and underwater tools system.

E-mail: zzm.zju@163.com

Ying-Long Chen, received the B.Eng. and Ph.D. degrees in mechatronic control engineering from Zhejiang University, Zhejiang, China, in 2008 and 2013, respectively. From 2013 to 2016, he has been a Research Assistant with School of Mechanical Engineering, Zhejiang University. Since 2017, he has been an Assistant Professor with the Naval Architecture and Ocean Engineering, Dalian Maritime University. His research interests include fluid power transmission and control, advanced motion control of mechatronic systems, and robotics.

E-mail: chenyinglong@dlmu.edu.cn

Yong-Jun Gong, received the Ph.D. degrees in mechatronic control engineering from Zhejiang University, Zhejiang, China, in 2005. He is currently an Professor with the Naval Architecture and Ocean Engineering College, Dalian Maritime University. His research interests include fluid power transmission and control, water hydraulics, and underwater tools system.

E-mail: DLMUwater@163.com

\section{Corresponding author: Zeng-Meng Zhang E-mail: zzm.zju@163.com}




\title{
Simulation Analysis and Experimental Research on Mechanical Properties of
}

\author{
Water-Hydraulic Ball Valve
}

Yong Yang ${ }^{1}$ Zeng-Meng Zhang ${ }^{1,2}$ • Ying-Long Chen ${ }^{1,3}$ • Yong-Jun Gong ${ }^{2,3}$

Received xxxx xx, 202x; revised $\mathrm{xxxx} \mathrm{xx}, 202 \mathrm{x}$; accepted $\mathrm{xxxx} \mathrm{xx}, 202 \mathrm{x}$

(C) Chinese Mechanical Engineering Society and Springer-Verlag Berlin Heidelberg 2017

Abstract: The working performance of a hydraulic system depends on the control valves, and the working performance of the control valves depends on their mechanical properties. Compared with the oil-hydraulic control valves, the water-hydraulic control valves are featured by strict sealing requirements, poor lubricity, and more complicated friction properties. In order to precisely control the valves, the fluid force and friction acting on the valve core are investigated through the methods of finite element analysis and experimental research. The results show that the realizable $\mathrm{k}-\varepsilon$ turbulence model with an appropriate wall roughness height could accurately reflect the state of fluid force. The fluid force increases as the valve opening and the pressure difference increase. Moreover, the friction is affected by the motion state of the valve core, and the reason is investigated. In addition, the phenomenon of mechanical properties variation is discussed, and an assumption is presented to explain the causation. This research can help understand some mechanical properties of water hydraulic valves and establish a more accurate mechanical

$\triangle$ Zeng-Meng Zhang zzm.zju@163.com

1 College of Naval Architecture and Ocean Engineering, Dalian Maritime University, China

2 Liaoning Provincial Key Laboratory of Rescue and Salvage Engineering, Dalian Maritime University, China

3 International Joint Research Center for Subsea Engineering Technology and Equipment, Dalian Maritime University, China model. Furthermore, the study also has guiding significance for controlling water hydraulic valves.

Keywords: Water-Hydraulic $・$ Ball valve $・$ Mechanical properties - CFD

\section{Introduction}

Compared with the traditional oil-hydraulic systems, the hydraulic medium of the water-hydraulic systems is pure water or seawater. The water-hydraulic systems have some advantages for the application on marine engineering. First, the water-hydraulic systems are compatible with the marine environment. The technology is environmentally friendly and safe for application on underwater equipment. For example, hydraulic systems generally have problems with hydraulic media leakage and discharge. When the problems occur, the oil-hydraulic systems often cause pollution and damage the marine environment [1]. However, the water-hydraulic systems could directly use seawater as the medium. Therefore, the leakage and discharge cause no pollution to the environment. On the other hand, the water-hydraulic system could ensure the safety of underwater construction, such as the rescue of the Kursk submarine. The water-hydraulic method, such as abrasive water jet, can open the sunken ship to prevent the hydraulic oil from burning and exploding. Second, the water-hydraulic systems have low frictional pressure loss and high long-distance transmission efficiency. Therefore, the power consumption of the water-hydraulic underwater 
working tools is lower than the oil-hydraulic. Third, the water-hydraulic systems of marine equipment need no pressure compensation. Therefore, the space and cost for the complicated and cumbersome oil tank and pressure compensation equipment are saved. Due to these advantages, the water-hydraulic system has also been widely used in other engineering practices $[2,3,4]$.

As the control valves are an essential part of the hydraulic system, the working performance of the hydraulic system depends on the working performance of the control valves [2]. Furthermore, the control valves' working performance depends on the valves' mechanical properties [3]. Therefore, the study of the mechanical properties of the water-hydraulic valves can guide the structural design of the valves and improve the working performance of the hydraulic systems. For example, one of the considering aspects of the design for the ship hydraulic system is reducing the negative impact on the hydraulic equipment caused by the sudden change of the hydraulic system [4]. This requires the hydraulic system to have a good control effect, which needs to master the control valves' mechanical properties. Therefore, this research focuses on the force acting on the valve core, mainly composed of the fluid force, the friction, and the spring force.

The fluid force is composed of the pressure force and the viscous force [5]. Compared with the oil-hydraulic valves, the structures of the water-hydraulic valves are more complicated, which results in the more complex flow fields in the valves [6]. The pressure distribution is not uniform in these complex flow fields, and the viscous force is tough to calculate through theoretical formulas directly. As a new type of calculation method, computational fluid dynamic (CFD) has the advantages of accuracy and convenience. In the turbulence flow field simulations, especially when the shape is complicated, the results' accuracy is related to the selection of turbulence models. The standard k- $\varepsilon$ model is valid only for the totally high Reynolds number flow fields. In many situations, the standard $\mathrm{k}-\varepsilon$ model can hardly provide enough accuracy [7]. However, some variant models of the standard k- $\varepsilon$ model, such as the realizable k- $\varepsilon$ model, can accurately analyze the low Reynolds number area of the flow fields [8]. Liang et al. [12] and Han et al. [13] investigated the cavitation phenomenon in a water-hydraulic valve through the CFD simulation based on the realizable $\mathrm{k}-\varepsilon$ turbulence model. Xu et al. [14] and Mlela et al. [15] optimized the structure of a water-hydraulic valve to decrease the erosion effect of cavitation through the CFD simulation. The RNG k- $\varepsilon$ turbulence model with $30 \mu \mathrm{m}$ wall roughness height was selected to conduct the simulation. Li et al. [16] used the
CFD method to calculate the fluid force acting on a nozzle flapper valve by the standard $\mathrm{k}-\varepsilon$ turbulence model. Moreover, Valdés et al. [10] considered that the standard $\mathrm{k}-\varepsilon$ turbulence model is valid only for totally high Reynolds number situations, and the RNG and realizable $\mathrm{k}-\varepsilon$ turbulence models could provide more accurate results by taking the effect of low Reynolds number area into account. They used the RNG k- $\varepsilon$ turbulence model to calculate the fluid force acting on a ball check valve. In order to improve the performance of a water-hydraulic valve, Han et al. [9] used the CFD method to analyze the fluid force acting on the valve core and optimize the valve core structure to improve the linearity of the valve. Based on the research, a new type of water-hydraulic proportional valve was designed, and the fluid force acting on the valve core was also calculated through the CFD simulation to optimize the structure of the proportional valve [9]. It can be seen that the CFD simulation can accurately analyze the flow field in the valve and perform a high-precision calculation of the fluid force.

Compared with hydraulic oil, the viscosity of water is low, which is prone to leakage [12,17]. Therefore, the water-hydraulic valves need stricter sealing than the oil-hydraulic valves. On the other hand, the insufficient lubrication of water leads to high friction of the water-hydraulic valves $[18,19]$. The friction generated by the sealing elements has a strong nonlinearity and depends on many factors, such as speed, compression of the seal ring. The bristle theory can explain this phenomenon well. Furthermore, Menga [20] studied the interaction between elastic coupling and friction. The results showed that compared with the no friction condition, the contact area between objects is larger when there is friction. Persson et al. [21] studied the friction process of rubber on smooth and rough substrates based on the theory of contact mechanics. In addition, the study also found that the contribution of the sources of friction is not the same at different speeds. Scaraggi et al. [22] investigated the lubricating sliding contact of nitrile rubber O-rings and steel surfaces with different surface roughness. Hong and Kim [23] improved the lubrication characteristic of the spool valve by using a spiral groove structure to make the valve core uniformly stressed. Meanwhile, the flow rate had also increased by using this structure. Zhang and Xie [24] researched the effect of water and hydraulic oil on the friction between the sealing rings and the steel through experiments. The sealing rings deformed during the movement of the valve cores, and the amount of deformation was different for different movement modes, and the friction changed accordingly. Because of the 
sealing elements and the insufficient lubrication of water, the mechanical properties of the water-hydraulic valves are more complex than the oil-hydraulic valves. Friction is an essential part of the mechanical properties of water hydraulic valves. Compared with traditional oil hydraulic systems, the friction of water hydraulic systems poses more challenges to fulfill the rapid and precise control. It is difficult to accurately describe the mechanical properties of the valves by considering only the fluid force or the sealing friction, respectively. At present, the research on this aspect needs more attention and more deeply analysis.

The ball valves have the advantages of simple structure, low cost, and good sealing performance [10]. Due to these advantages, the ball valves are widely used in pipeline transportation, automotive engineering, marine exploration, and medicine [10,26,27,28]. Ferreira et al. [29] studied the performance of the ball valves used in pipeline flow control. Ma et al. [30] researched the control effect of the ball valves on the gas-liquid two-phase flow. Cui et al. [31] analyzed the influence of the opening and closing process of the ball valves on the performance of the hydraulic system. As a typical control valve structure, the dynamic research of the water-hydraulic ball valves has guiding significance for the design and application of the water-hydraulic valves.

This article uses the realizable $\mathrm{k}-\varepsilon$ turbulence models with different wall roughness heights to simulate and analyze the fluid force acting on the ball valve core. The models' accuracies are compared, and the reason for the generation and variation of the fluid force is discussed. Based on a comprehensive water-hydraulic system experimental platform, four different test methods are used to conduct the ball valve mechanical experiments, and the influence of the movement mode on the friction is investigated. The results are analyzed based on the bristle model. In addition, the phenomenon of mechanical properties variation is discussed, and an assumption is presented to explain the causation. Moreover, the assumption is verified by the finite element simulation.

\section{Simulation Analysis}

\subsection{Working Principle of Water-Hydraulic Ball Valve}

The water-hydraulic ball valve is composed of a valve body, a valve core, valve sleeves, a spring, sealing rings, and a displacement sensor, as shown in Figure 1. When the ball valve is working, the water flows into the P-port and flows out from the T-port, and the water pressure decreases by the throttling effect of the flow resistance. The flow resistance depends on the valve port's opening degree controlled by the pushrods. Moreover, the displacement of the pushrods is measured by the displacement sensor.

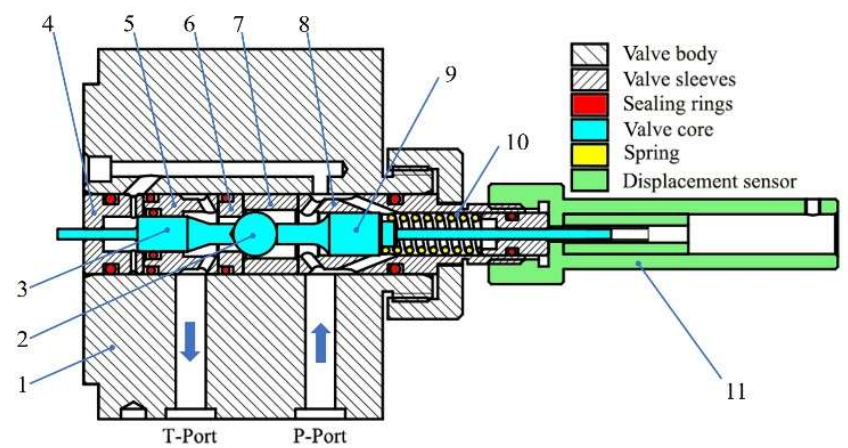

Figure 1 Schematic diagram of the water-hydraulic ball valve (1) valve body, (2) ball valve core, (3) output pushrod, (4)

equilibrating valve sleeve, (5) output valve sleeve, (6) valve seat,

(7) connecting valve sleeve, (8) input valve sleeve, (9) input pushrod, (10) spring, (11) displacement sensor

Link pipelines connect both sides end chambers and the chamber linking the P-port, and the water in these chambers forms a high-pressure region. Moreover, the water in the chamber linking the T-port forms a low-pressure region. Because the sum of the axial projection area of the high-pressure region is zero, and the sum of the low-pressure region is zero, the liquid pressure force in the axial direction is zero when the pressures of the high-pressure region and the low-pressure region are constants.

The sealing rings are installed in the valve to avoid leakage. In the right chamber, a spring is installed to provide a force in the valve port closing direction, which is used to close and fasten the valve core. The main parameters of the ball valve are shown in Table 1. Moreover, Figure 2 shows the valve core and the valve sleeves.

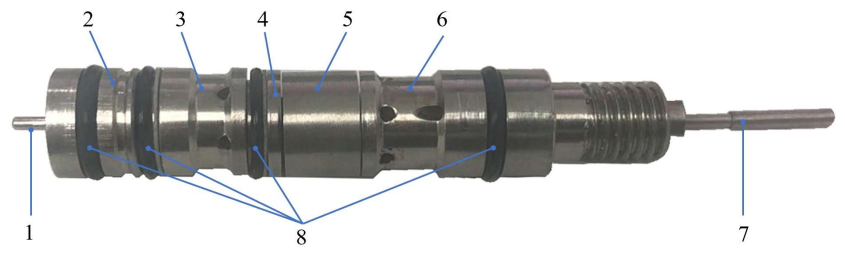

Figure 2 Valve core and valve sleeves (1) output pushrod, (2) equilibrating valve sleeve, (3) output valve sleeve, (4) valve seat, (5) connecting valve sleeve, (6) input valve sleeve, (7) input pushrod, (8) sealing rings 
Table 1 Main parameters of the ball valve

\begin{tabular}{ll}
\hline Items & Parameters \\
\hline Diameter of the ball valve core $(\mathrm{mm})$ & 8.73 \\
Diameter of the throttle base $(\mathrm{mm})$ & 7 \\
Chamfer of the throttle base & $0.5 \mathrm{~mm} \times 45^{\circ}$ \\
Spring stiffness coefficient $(\mathrm{N} / \mathrm{mm})$ & 25 \\
Rated valve opening $(\mu \mathrm{m})$ & 300 \\
Rated working pressure $(\mathrm{MPa})$ & 3 \\
\hline
\end{tabular}

\subsection{Simulation Parameters}

The simulation model of the ball valve is established in the finite element simulation software, as shown in Figure 3. The two-dimensional axisymmetric model is selected to decrease the working load of the simulations. Because the shape of the flow field is complicated and there are some low Reynolds number regions in the flow field, the realizable $\mathrm{k}-\varepsilon$ turbulence model with different wall roughness heights is selected to conduct the simulations. Table 2 shows the properties of the hydraulic medium. Moreover, a total of 120 simulation calculations with four different wall roughness heights, three pressure differences and ten different valve openings are performed, as shown in Table 3. Figure 4 shows the mesh model when the valve opening is $150 \mu \mathrm{m}$, containing 30442 domain elements and 1854 boundary elements.

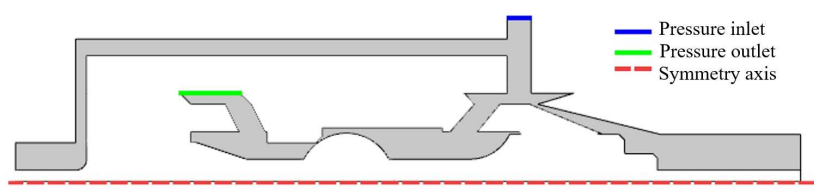

Figure 3 Simulation model of the ball valve

Table 2 Material properties for water medium

\begin{tabular}{ll}
\hline Items & Parameters \\
\hline Temperature $(\mathrm{K})$ & 293.15 \\
Density $(\mathrm{kg} / \mathrm{m} 3)$ & 998.2 \\
Viscosity $(\mathrm{Pa} \cdot \mathrm{s})$ & $1.002 \times 10-3$ \\
\hline
\end{tabular}

Table 3 Simulation conditions

\begin{tabular}{ll}
\hline Simulation conditions & Parameters \\
\hline Inlet pressure $(\mathrm{MPa})$ & $1.5,2.5,3.5$ \\
Outlet pressure $(\mathrm{MPa})$ & 0.5 \\
Wall roughness height $(\mu \mathrm{m})$ & $0,10,20,30$ \\
Valve opening $(\mu \mathrm{m})$ & $30,60,90,120,150,180,210$, \\
& $240,270,300$ \\
\hline
\end{tabular}

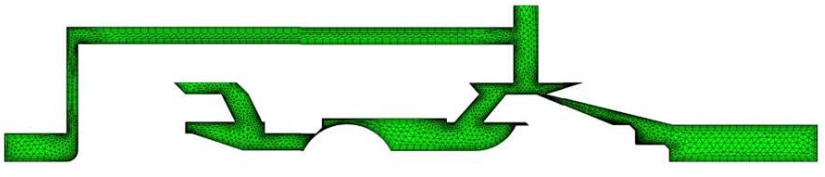

Figure 4 Mesh model of the simulation when the valve opening is $150 \mu \mathrm{m}$

\subsection{Simulation Results}

When the opening is $150 \mu \mathrm{m}$, and the pressure difference is $3 \mathrm{MPa}$, the velocity cloud picture and pressure cloud picture are shown in Figure 5. It can be found that the water pressure around the orifice changes with the variation of the velocity. The orifice divides the flow field into a high-pressure region and a low-pressure region. The maximum velocity appears where the flow area is the smallest, and the velocity decreases after it flows out of the orifice. It is worth noting that there is a high-speed jet behind the orifice, and the jet impacts the pushrod. On the other hand, there are dead zones and vortexes in the flow field. These phenomena result in the uneven variation of the water pressure, which causes the variation of the liquid force.

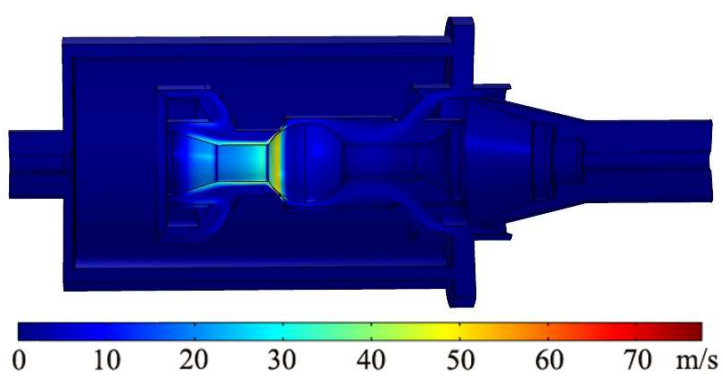

(a)

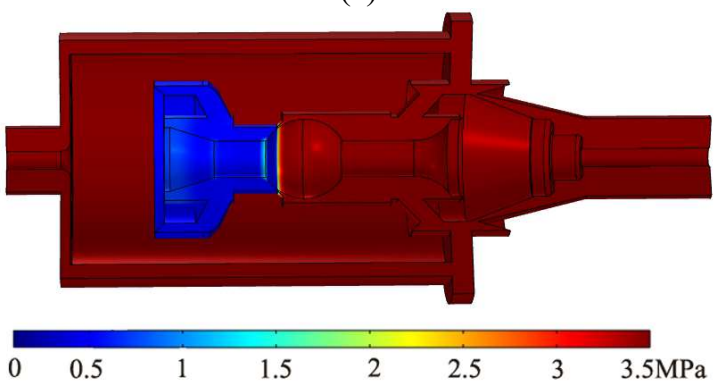

(b)

Figure 5 Simulation flow field, (a) velocity cloud picture and (b) pressure cloud picture

Since the control object is the ball valve core, the fluid force acting on the valve core is calculated. The fluid force consists of two parts: the pressure force and the viscous force. The post-processing functions of the software, including the surface integral and the turbulence parameters 
calculation, are used to calculate the fluid force. The pressure force can be calculated by Eq. (1):

$$
F_{\mathrm{s}}=\int p \cdot n_{\mathrm{z}} d A
$$

where $F_{\mathrm{s}}$ is the pressure force, $\mathrm{p}$ is the pressure, $n_{\mathrm{z}}$ is the axial projection, and $A$ is the surface area of the valve core.

The viscous force acting on the valve core can be calculated by Eq. (2):

$$
F_{\mathrm{v}}=\int s_{\mathrm{z}} d A
$$

where $F_{\mathrm{v}}$ is the viscous force, $s_{\mathrm{z}}$ is the axial viscous strength.

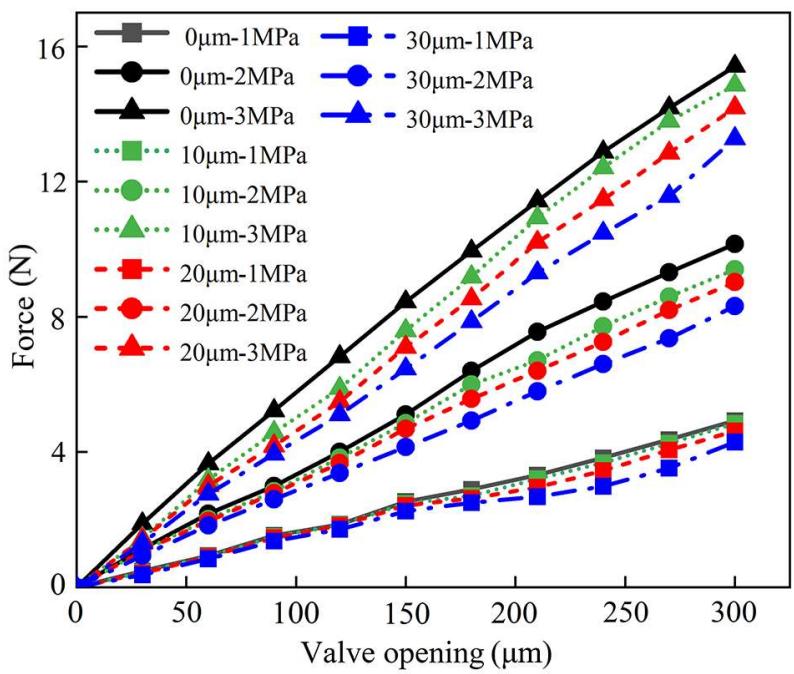

Figure 6 Simulation fluid forces of different wall roughness heights

Figure 6 shows the simulation results of fluid force. It can be seen from Figure 6 that when the pressure difference is $2 \mathrm{MPa}$ and the opening is $150 \mu \mathrm{m}$, from $0 \mu \mathrm{m}$ to $30 \mu \mathrm{m}$ wall roughness heights, the fluid forces are $5.1 \mathrm{~N}, 4.9 \mathrm{~N}$, $4.7 \mathrm{~N}$, and $4.2 \mathrm{~N}$. When the pressure difference is $2 \mathrm{MPa}$, and the opening is $300 \mu \mathrm{m}$, the forces are $10.2 \mathrm{~N}, 9.4 \mathrm{~N}, 9$ $\mathrm{N}$, and $8.3 \mathrm{~N}$. When the pressure difference is $3 \mathrm{MPa}$, and the opening is $150 \mu \mathrm{m}$, the values are $8.4 \mathrm{~N}, 7.6 \mathrm{~N}, 7.1 \mathrm{~N}$, and $6.5 \mathrm{~N}$. Moreover, when the pressure difference is 3 $\mathrm{MPa}$, and the opening is $300 \mu \mathrm{m}$, the values are $15.4 \mathrm{~N}$, 14.9 N, 14.2 N, and 13.3 N. It can be found that the fluid force increases as the valve opening and the pressure difference increase. Furthermore, the fluid force decreases as the wall roughness height increases.

\section{Mechanical Experiments}

\subsection{Experimental Equipment}

The comprehensive water-hydraulic system experimental platform comprises a water-hydraulic pump, a display system, a tested valve, and connecting pipelines, as shown in Figure 7. Figure 8 shows the tested ball valve composed of a micrometer with a self-locking function, a ball valve, a force sensor, and a displacement sensor. The sensors measure the force and opening. Furthermore, the display system, including a computer with Labview software and a National Instruments capture card, shows the measured signals. The measure ranges of the force and displacement sensors are $\pm 100 \mathrm{~N}$ and $\pm 2.5 \mathrm{~mm}$, and the nonlinear errors are less than $0.5 \%$ FRO.
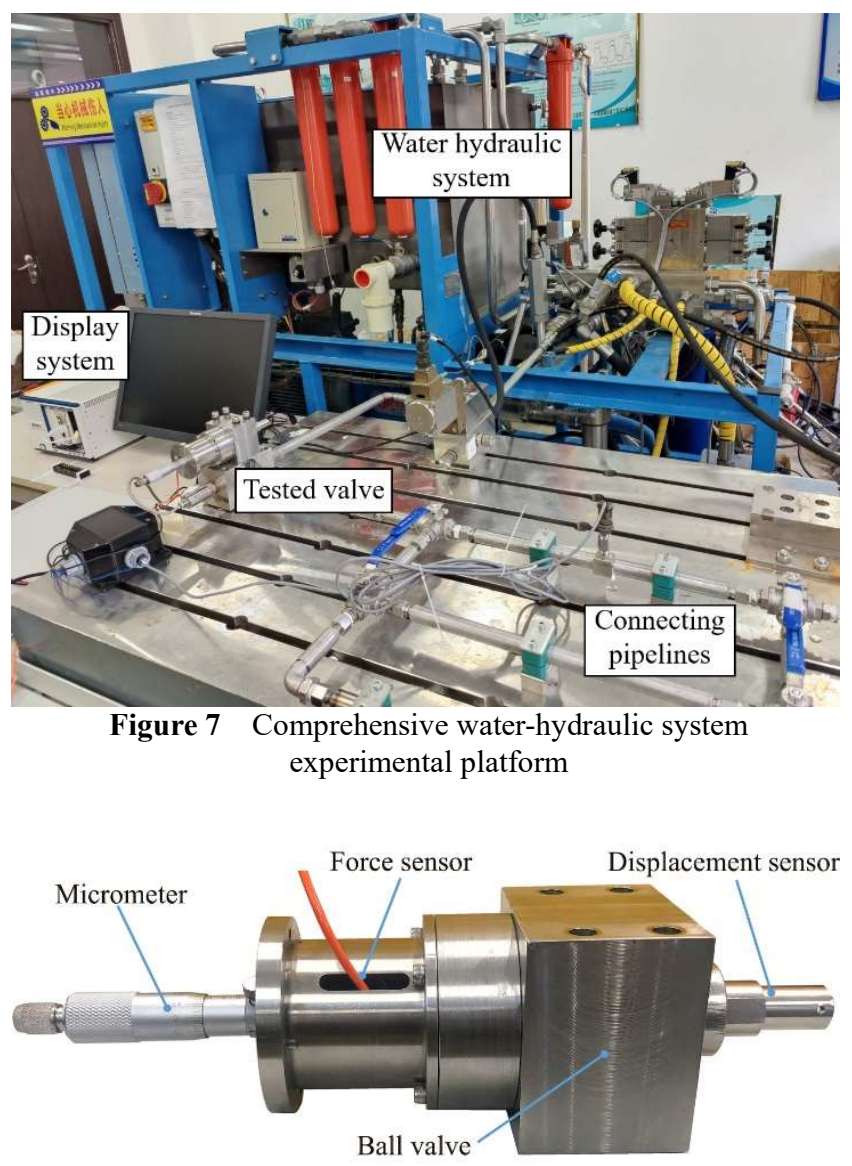

Figure 8 Tested ball valve

\subsection{Experimental Methods}

Considering that the ball valve's force state is complicated, to explore the mechanical properties accurately, the water-hydraulic system with a pressure difference of $3 \mathrm{MPa}$ is taken as the research object, and four different test methods are used to conduct the experiments. For the convenience of reading, the points of 
$30 \mu \mathrm{m}, 60 \mu \mathrm{m}, 90 \mu \mathrm{m}, 120 \mu \mathrm{m}, 150 \mu \mathrm{m}, 180 \mu \mathrm{m}, 210 \mu \mathrm{m}$, $240 \mu \mathrm{m}, 270 \mu \mathrm{m}$, and $300 \mu \mathrm{m}$ openings are regarded as the measuring points. Moreover, the opening increasing direction is regarded as the positive direction. Here are the descriptions of the four test methods.

(1) Move the valve core in the positive direction at a low speed. Moreover, record the force sensor data when the valve core passes the measuring points.

(2) Move the valve core to each measuring point and stop until the force sensor data is stable. Record the stable data of each measuring point.

(3) First, move the valve core in the positive direction and stop when the valve core exceeds a measuring point of about $3 \mu \mathrm{m}$. Second, move the valve core a short distance opposite, covering the measuring point. When the valve core passes the measuring point in the opposite direction, the force sensor data is recorded. Moreover, the following measuring points are operated in the same way until the last measuring point is completed.

(4) First, move the valve core to $500 \mu \mathrm{m}$. Second, continuously move the valve core in the opposite direction, such as the test method 1. Finally, record the force sensor data when the valve core passes the measuring points.

\subsection{Experimental Results}

In order to facilitate understanding, the measured data is processed to eliminate the spring influence, including the pre-tightening force and elastic force caused by valve core moving. Moreover, different from the previous, the valve closing direction is regarded as positive. Figure 9 shows the experimental results.

It can be seen that as the opening increases, the sum of the liquid force and the friction increases. It is worth noting that the resultant forces measured under different test methods are different. When the opening is $150 \mu \mathrm{m}$, the values of the resultant forces from method 1 to method 4 are $6.8 \mathrm{~N}, 5.2 \mathrm{~N}, 1.2 \mathrm{~N}$, and $-4.8 \mathrm{~N}$, respectively. When the opening is $300 \mu \mathrm{m}$, the values are $13.6 \mathrm{~N}, 12 \mathrm{~N}, 8.7 \mathrm{~N}$, and $2.6 \mathrm{~N}$.

It can be found from the comparison of method 1 and method 2 that the forces when the valve core is continuously moving and stable are different. Furthermore, the difference between the two methods is about $1 \mathrm{~N}$ to $2 \mathrm{~N}$. Considering the data of method 3 and method 4 , it can be known that the force state of the valve core is affected by the movement direction and the movement mode. The force is the largest when the valve core continuously moves in the valve opening direction. Furthermore, the force is the smallest when the valve core continuously moves in the reverse direction.

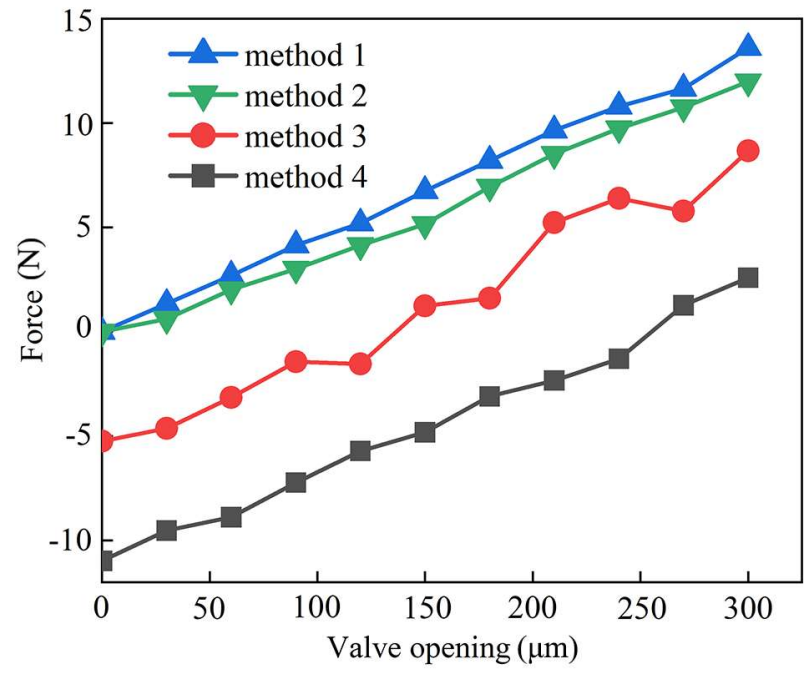

Figure 9 Measured data of the four different test methods

\section{Discussion}

The force acting on the valve core comprises the spring force, liquid force and friction. The effect of the spring force is eliminated in this research. The liquid force is caused by the uneven pressure change in the flow field and the viscous drag effect of water on the valve core.

From the experimental results, it can be known that the friction is affected by the motion state of the valve core. When the motion states are continuously moving and steady, the frictions are different. Furthermore, when the valve core moves in the reverse direction, the friction does not change instantaneously but increases with the increase of the reverse displacement. The bristle theory can explain this phenomenon. The friction source is regarded as the bristles on surfaces, and the deformation amount of the bristles determines the friction. Based on the bristle theory, many friction phenomena have been properly explained, and some friction models, such as the Lugre friction model, are developed. These models have a wide range of uses.

Specifically, when the valve core moves in the valve opening direction continuously, the bristles reach the maximum deformation state at this speed after a certain displacement, and the friction reaches the maximum state. When the direction changes, the friction changes from the maximum state in one direction to the maximum state in the other direction. Nevertheless, the process cannot 
finish immediately because the bristles deformation cannot be completed instantly. The conversion process requires a certain amount of displacement to complete. The friction is between the two maximum states when the displacement changes less than the required value. And this is the reason for the difference between the values of the test methods 3 and 4 . On the other hand, the reason for the friction change after the valve core stops is that the hardness of the sealing rings is low, and it is prone to elastic deformation or creep. When the valve core stops moving, the sealing rings recover, and the bristles on the sealing rings also recover. The displacement of the bristles reduces the deformation of the bristles, which in turn reduces the friction. However, the amount of the elastic deformation is limited, so the friction reduces after the valve core stops but not to zero. This phenomenon makes the method of the friction calculating based on the valve core displacement and velocity no longer applicable. Instead, it is necessary to add a time term or a parameter about the elastic deformation to describe the sealing rings' friction accurately. On the other hand, this phenomenon also impacts the control method of the water hydraulic valves, especially when the valves stop moving and then reopen this movement.

Figure 10 shows the measured force and valve opening curves when the valve core stops after motion and veers. The stage $\mathrm{A}$ to $\mathrm{B}$ is the bristles elastic deformation stage after standing. The stage $\mathrm{B}$ to $\mathrm{C}$ is the deformation recovery stage. In the end, the bristles deform to the maximum state, and the friction hardly changes. This phenomenon can be summarized as when the motion state changes, the friction changes. After the valve core stops, the friction reduces by a certain amount. And when the movement direction changes, the friction acting on the valve core is related to the displacement change. Within a certain range, the greater the displacement changes, the greater the friction changes.

As the friction is related to the motion state of the valve core, when the valve core is continuously moving at a low speed, such as the test method 1 , the motion state can be approximately regarded as constant, and the friction is also approximately a constant value. Therefore, the processed test method 1 data can be regarded as the value of the liquid force without friction interference. And test method 1 can be used to measure the liquid force acting on the valve core.

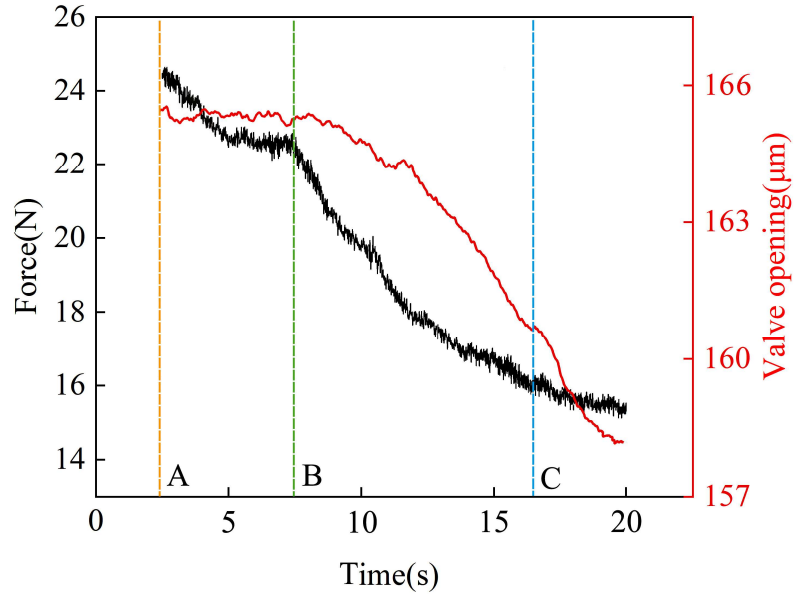

Figure 10 The measured force and valve opening curves
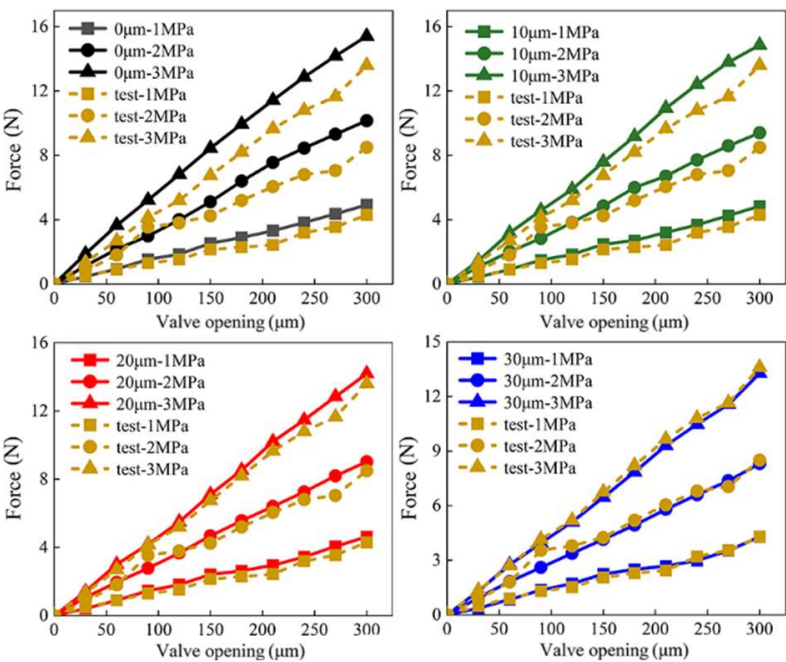

Figure 11 Liquid forces of the simulations and the experiments

The liquid forces of the simulations with different models and the experiments are shown in Figure 11. It can be known that the liquid force increases as the valve opening and the pressure difference increase. When the valve opening is $150 \mu \mathrm{m}$, and the pressure difference is 3 $\mathrm{MPa}$, the liquid forces of the experiment and the no wall roughness model are $6.7 \mathrm{~N}$ and $8.4 \mathrm{~N}$, and the deviation is $25.4 \%$. The liquid force of the $10 \mu \mathrm{m}$ wall roughness height model is $7.6 \mathrm{~N}$, and the deviation of the model and the experiment is $13.4 \%$. The liquid force of the $20 \mu \mathrm{m}$ wall roughness height model is $7.1 \mathrm{~N}$, and the deviation is $6 \%$. The liquid force of the $30 \mu \mathrm{m}$ wall roughness height model is $6.5 \mathrm{~N}$, and the deviation is $3 \%$. Furthermore, when the valve opening is $300 \mu \mathrm{m}$, and the pressure difference is 3 
$\mathrm{MPa}$, the liquid forces of the experiment and the no wall roughness model are $13.6 \mathrm{~N}$ and $15.4 \mathrm{~N}$, and the deviation is $13.2 \%$. The liquid force of the $10 \mu \mathrm{m}$ wall roughness height model is $14.9 \mathrm{~N}$, and the deviation of the model and the experiment is $9.6 \%$. The liquid force of the $20 \mu \mathrm{m}$ wall roughness height model is $14.2 \mathrm{~N}$, and the deviation is $4.2 \%$. The liquid force of the $30 \mu \mathrm{m}$ wall roughness height model is $13.3 \mathrm{~N}$, and the deviation is $2.2 \%$. It can be found that the realizable $\mathrm{k}-\varepsilon$ turbulence model with $30 \mu \mathrm{m}$ wall roughness height is closest to the reality.

It is worth noting that the connection method of the pushrods and the ball valve core is not fixed but linked by the press effect of the external forces. Due to the unstable connection method, the ball valve core may deflect and deviate from the central axis during the movement, as shown in Figure 12. The deviation of the ball valve core would cause the change of the flow field in the valve and then affect the liquid force acting on the valve core. Furthermore, a CFD analysis is carried out to investigate the effect of the deviation on the liquid force.

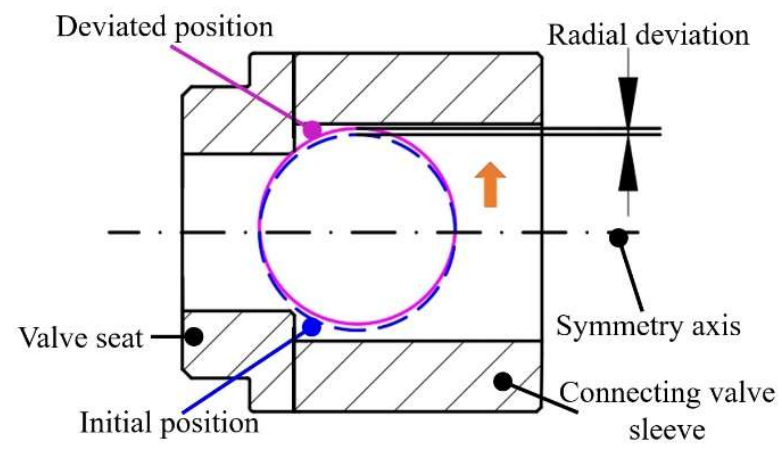

Figure 12 Radial deviation of the ball valve core

Table 4 Parameters of the valve core deviation simulations

\begin{tabular}{cccccc}
\hline $\begin{array}{c}\text { Opening } \\
(\mu \mathrm{m})\end{array}$ & Case 1 & Case 2 & Case 3 & Case 4 & Case 5 \\
\hline 90 & 0 & 20 & 40 & 60 & 80 \\
150 & 0 & 30 & 60 & 90 & 120 \\
210 & 0 & 50 & 100 & 150 & 200 \\
270 & 0 & 60 & 120 & 180 & 240 \\
\hline
\end{tabular}

Table 4 shows the parameters of the simulations. Same as the preceding simulations, the medium is water, and the pressure differences are $1 \mathrm{MPa}, 2 \mathrm{MPa}$, and $3 \mathrm{MPa}$. Since the physical model is no longer axisymmetric, the three-dimensional physical model is selected. Based on the previous analysis, the realizable $\mathrm{k}-\varepsilon$ turbulence model with $30 \mu \mathrm{m}$ wall roughness height is selected to conduct the simulations. Moreover, the preceding CFD results show no liquid flowing in the two end chambers, and the pressure in the chambers is stable and constant. Therefore, only the middle part of the flow field is simulated to simplify the simulations.

Taking the pressure difference of $3 \mathrm{MPa}$, the valve opening of $150 \mu \mathrm{m}$, and the radial deviation of $120 \mu \mathrm{m}$ as an example, the flow field simulation result is shown in Figure 13. It can be seen that the radial deviation of the ball valve core affects the flow field state and changes the flow distribution of the field.

Figure 14 shows the results of the simulations, taking the results when the ball valve core has no deviation as the zero points and taking the valve closing direction as the positive direction. It can be seen that the radial deviation of the ball valve core affects the liquid force. However, the effect is to increase or decrease the force, and the effect degree is affected by the valve opening, the pressure difference, and the degree of the valve core deviation. There is no strong regularity in the effect of the radial deviation on the liquid force. For example, in the case of $150 \mu \mathrm{m}$ opening and 3 $\mathrm{MPa}$ pressure difference, when the radial deviation is 30 $\mu \mathrm{m}$, the effect increases the liquid force by $0.39 \mathrm{~N}$. When the radial deviation is $120 \mu \mathrm{m}$, the effect decreases the liquid force by $2.36 \mathrm{~N}$.

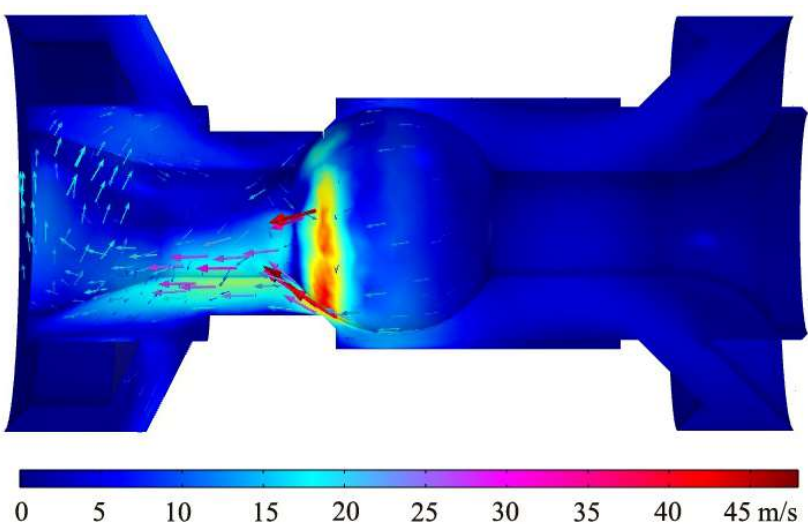

Figure 13 Flow field simulation result of the simulation

The phenomenon that the unstable connection causes the liquid force change is also reflected in the experiments. Figure 15 shows the experimental data when the pressure difference is $2 \mathrm{MPa}$, and the valve core moves continuously in the valve opening direction. When the valve openings are $90 \mu \mathrm{m}$ and $270 \mu \mathrm{m}$, the continuity of the liquid force result is unsatisfactory. This may be due to the radial deviation of the ball valve core.

As shown in Figure 16, the averages of the measured values of the adjacent measuring points are regarded as the expected liquid forces of $90 \mu \mathrm{m}$ and $270 \mu \mathrm{m}$ valve openings. Moreover, the difference between the actual measured and expected values is regarded as the liquid force change caused by the radial deviation. Since the 
amount of the change is within the range of simulation results, the theory of radial deviation can explain the cause of this phenomenon.

According to the experimental results that the liquid force values generally have good continuity, it can be found that the probability of valve core deviation is small, and the impact is limited. The effect on the entire mechanical properties is within an acceptable range.
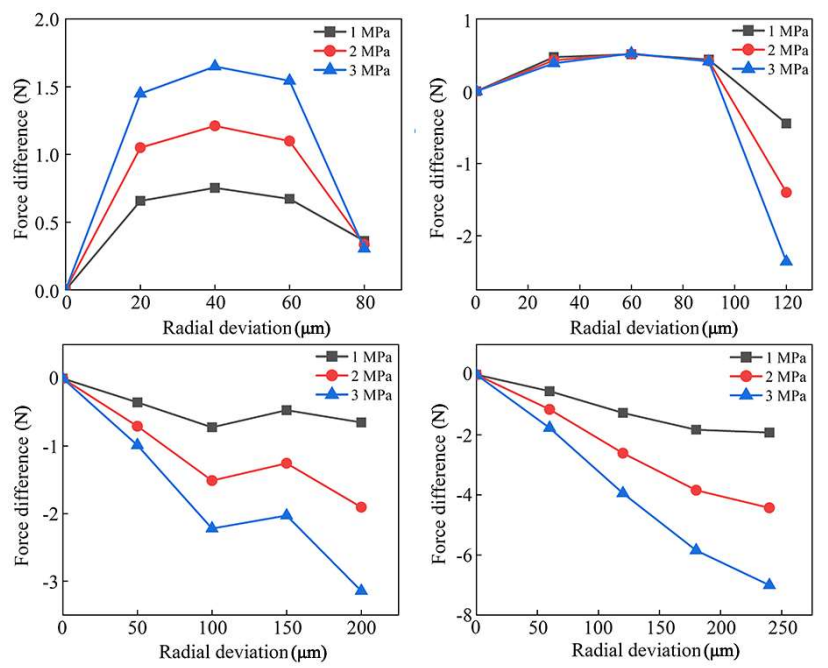

Figure 14 Force results of the simulations, (a) $90 \mu \mathrm{m}$ opening, (b) $150 \mu \mathrm{m}$ opening, (c) $210 \mu \mathrm{m}$ opening, (d) $270 \mu \mathrm{m}$ opening

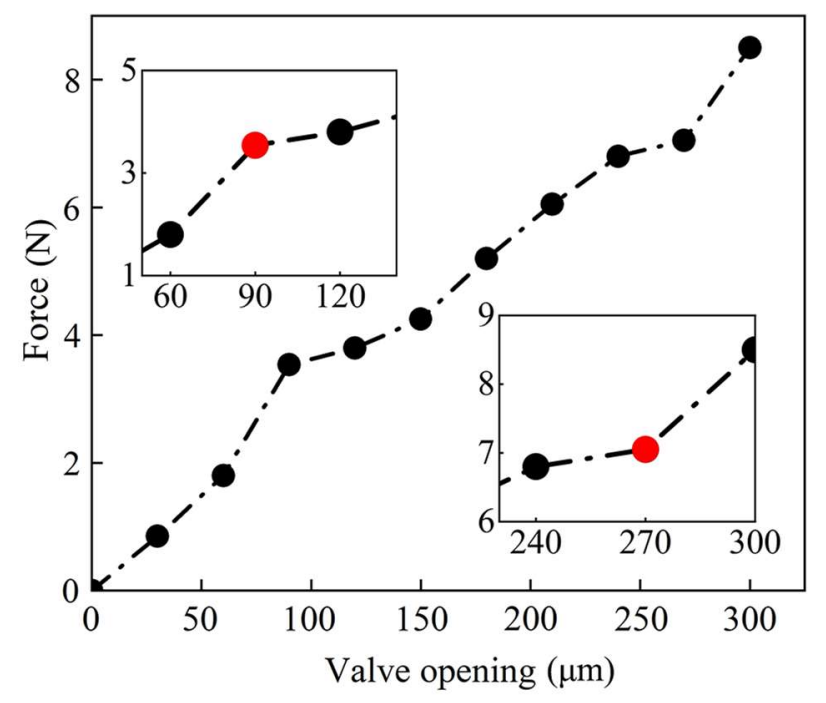

Figure 15 Experimental data when the pressure difference is $2 \mathrm{MPa}$ in test method 1

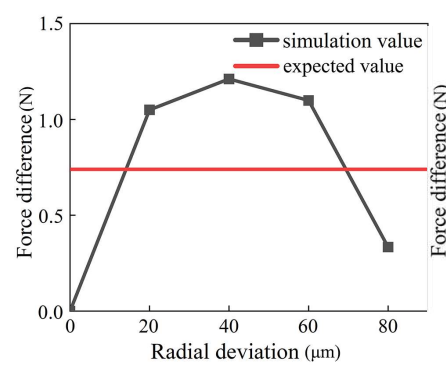

(a)

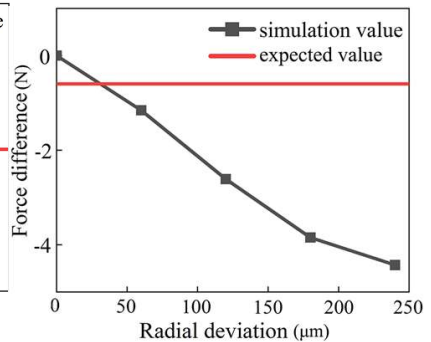

(b)
Figure 16 The expected values and the simulation values, (a) $90 \mu \mathrm{m}$ opening, (b) $270 \mu \mathrm{m}$ opening

\section{Conclusion}

Through the simulations and the experiments, the mechanical properties of the water-hydraulic ball valve are analyzed. Here are the conclusions about the characteristics.

The liquid force acting on the valve core is caused by the uneven pressure change in the flow field and the medium viscous drag effect. The liquid force increases as the valve opening and the pressure difference increase. Compared with the other wall roughness heights realizable $\mathrm{k}-\varepsilon$ turbulence models, the $30 \mu \mathrm{m}$ wall roughness height model is closest to the reality. Furthermore, when the pressure difference is $3 \mathrm{MPa}$, and the opening is $300 \mu \mathrm{m}$, the deviation is $2.2 \%$.

The friction acting on the valve core is affected by the motion state of the valve core. The friction values when the motion states are continuously moving and stable are different. This phenomenon leads to the need for a more accurate friction model and corresponding control strategies for water hydraulic valves. Furthermore, within a certain range, the greater the valve core displacement changes, the greater the friction changes.

The unstable connection between the ball valve core and pushrods could cause variations in the mechanical properties. Nevertheless, the probability of this phenomenon is small, and the impact is limited. The influence on the entire mechanical properties is still within an acceptable range. 


\section{Declaration}

\author{
Availability of data and materials \\ Not applicable.
}

\section{Competing interests}

The authors declare no competing financial interests.

\section{Funding}

This work was supported by the National Natural Science Foundation of China (Grant No. 52175043 and U908228), the Natural Science Foundation of Liaoning Province (Grant No. 2020-HYLH-18), the Science and Technology Innovation Fund Project of Dalian (Grant No. 2019J12GX041), and the Fundamental Research Funds for the Central Universities of China.

\section{Authors' contributions}

Not applicable.

\section{Acknowledgements}

Not applicable.

\section{References}

[1] L Yang, C T Lai. Biological treatment of mineral oil in a salty environment. Water Science and Technology, 2000, 42(7-8): 369-375.

[2] Z Zhang, J Hou, D Ning, et al. Modeling and experiments on the drive characteristics of high-strength water hydraulic artificial muscles. Smart Materials and Structures, 2017, 26(5).

[3] H Zhou. Water-Assisted Injection Molding System Based on Water Hydraulic Proportional Control Technique. Chinese Journal of Mechanical Engineering, 2010, 23(4): 418-427.

[4] C Wang, G Xia, T Cong, et al. Operation characteristics analyses on a marine-type passive residual heat removal system. Annals of Nuclear Energy, 2018, 120: 546-558.

[5] C Lv, H Wang,D Cao. High-Precision Hydraulic Pressure Control Based on Linear Pressure-Drop Modulation in Valve Critical Equilibrium State. IEEE Transactions on Industrial Electronics, 2017, 64(10): 7984-7993.

[6] L Baoren, G Longlong, Y Gang. Modeling and Control of a Novel High-Pressure Pneumatic Servo Valve Direct-Driven by Voice Coil Motor. Journal of Dynamic Systems, Measurement, and Control, 2013, 135(1): 1-5.

[7] K Urbanowicz. Modern Modeling of Water Hammer. Polish Maritime Research, 2017, 24(3): 68-77.

[8] E Lisowski, W Czyżycki,J Rajda. Three dimensional CFD analysis and experimental test of flow force acting on the spool of solenoid operated directional control valve. Energy Conversion and Management, 2013, 70: 220-229.

[9] M Han, Y Liu, D Wu, et al. Numerical Analysis and Optimisation of the Flow Forces in a Water Hydraulic Proportional Cartridge Valve for Injection System. Ieee Access, 2018, 6: 10392-10401.

[10] J R Valdés, J M Rodríguez, R Monge, et al. Numerical simulation and experimental validation of the cavitating flow through a ball check valve. Energy Conversion and Management, 2014, 78: 776-786.

[11] T H Shih, W W Liou, A Shabbir, et al. A new k- $\epsilon$ eddy viscosity model for high reynolds number turbulent flows. Computers and Fluids, 1994, 24(3): 227-238.

[12] J Liang, X Luo, Y Liu, et al. A numerical investigation in effects of inlet pressure fluctuations on the flow and cavitation characteristics inside water hydraulic poppet valves. International Journal of Heat and Mass Transfer, 2016, 103: 684-700.

[13] M Han, Y Liu, D Wu, et al. A numerical investigation in characteristics of flow force under cavitation state inside the water hydraulic poppet valves. International Journal of Heat and Mass Transfer, 2017, 111: 1-16.

[14] $\mathrm{H} \mathrm{Xu}, \mathrm{H}$ Wang, M Hu, et al. Optimal Design and Experimental Research of the Anti-Cavitation Structure in the Water Hydraulic Relief Valve. Journal of Pressure Vessel Technology, 2018, 140(5): $1-8$.

[15] M K Mlela, H Xu, F Sun, et al. Material Analysis and Molecular Dynamics Simulation for Cavitation Erosion and Corrosion Suppression in Water Hydraulic Valves. Materials (Basel), 2020, 13(2).

[16] L Li, H Yan, H Zhang, et al. Numerical simulation and experimental research of the flow force and forced vibration in the nozzle-flapper valve. Mechanical Systems and Signal Processing, 2018, 99: 550-566.

[17] M Han, Y Liu, K Zheng, et al. Investigation on the modeling and dynamic characteristics of a fast-response and large-flow water hydraulic proportional cartridge valve. Proceedings of the Institution of Mechanical Engineers, Part C: Journal of Mechanical Engineering Science, 2020, 234(22): 4415-4432.

[18] F Majdic,J Pezdirnik. Oil - and water - based continuous control valve. Industrial Lubrication and Tribology, 2010, 62(3): 136-143.

[19] F Majdič, J Pezdirnik,M Kalin. Experimental validation of the lifetime performance of a proportional $4 / 3$ hydraulic valve operating in water. Tribology International, 2011, 44(12): 2013-2021.

[20] N Menga. Rough frictional contact of elastic thin layers: The effect of geometrical coupling. International Journal of Solids and Structures, 2019, 164: 212-220.

[21] B N J Persson, B Lorenz, M Shimizu, et al. Multiscale contact mechanics with application to seals and rubber friction on dry and lubricated surfaces. Advances in Polymer Science, 2017, 275(August 2016): 103-156.

[22] M Scaraggi, J Angerhausen, L Dorogin, et al. Influence of anisotropic surface roughness on lubricated rubber friction: Extended theory and an application to hydraulic seals. Wear, 2018, 410-411: 43-62.

[23] S H Hong,K W Kim. A new type groove for hydraulic spool valve. Tribology International, 2016, 103: 629-640.

[24] J Zhang,J Xie. Investigation of Static and Dynamic Seal Performances of a Rubber O-Ring. Journal of Tribology, 2018, 140(4): 1-11.

[25] A S Tabrizi, M Asadi, G Xie, et al. Computational fluid-dynamics-based analysis of a ball valve performance in the presence of cavitation. Journal of Engineering Thermophysics, 2014, 23(1): 27-38.

[26] J Tao, Z Lin, C Ma, et al. An Experimental and Numerical Study of Regulating Performance and Flow Loss in a V-Port Ball Valve. Journal of Fluids Engineering, 2020, 142(2): 1-9.

[27] S C Morgan, A D Hendricks, M L Seto, et al. A Magnetically Tunable Check Valve Applied to a Lab-on-Chip Nitrite Sensor. 
Sensors (Basel), 2019, 19(21).

[28] X Jiang, J C Loeb, C Manzanas, et al. Valve-Enabled Sample Preparation and RNA Amplification in a Coffee Mug for Zika Virus Detection. Angew Chem Int Ed Engl, 2018, 57(52): 17211-17214.

[29] J P B C C Ferreira, N M C Martins,D I C Covas. Ball Valve Behavior under Steady and Unsteady Conditions. Journal of Hydraulic Engineering, 2018, 144(4).

[30] Y Ma, M Zhang,H Luo. Numerical and experimental studies of gas-liquid flow and pressure drop in multiphase pump valves. Science progress, 2020, 103(3): 1-25.

[31] B Cui, Z Lin, Z Zhu, et al. Influence of opening and closing process of ball valve on external performance and internal flow characteristics. Experimental Thermal and Fluid Science, 2017, 80: 193-202.

\section{Biographical notes}

Yong Yang, received the B.Eng. degrees in mechanism design, manufacturing and automatization from Dalian Maritime University, Dalian, China, in 2018. Since 2018, he has been a master student with the Naval Architecture and Ocean Engineering College, Dalian Maritime University. His research interests include fluid power transmission and control, water hydraulics and control valves.

E-mail: dlmu_yy@163.com

Zeng-Meng Zhang, received the Ph.D. degrees in mechatronic control engineering from Zhejiang University, Zhejiang, China, in 2009. He is currently an Professor with the Naval Architecture and Ocean Engineering College, Dalian Maritime University. His research interests include fluid power transmission and control, water hydraulics, artificial muscles, control valves and underwater tools system.

E-mail: zzm.zju@163.com

Ying-Long Chen, received the B.Eng. and Ph.D. degrees in mechatronic control engineering from Zhejiang University, Zhejiang, China, in 2008 and 2013, respectively. From 2013 to 2016, he has been a Research Assistant with School of Mechanical Engineering, Zhejiang University. Since 2017, he has been an Assistant Professor with the Naval Architecture and Ocean Engineering, Dalian Maritime University. His research interests include fluid power transmission and control, advanced motion control of mechatronic systems, and robotics.

E-mail: chenyinglong@dlmu.edu.cn

Yong-Jun Gong, received the Ph.D. degrees in mechatronic control engineering from Zhejiang University, Zhejiang, China, in 2005. He is currently an Professor with the Naval Architecture and Ocean Engineering College, Dalian Maritime
University. His research interests include fluid power transmission and control, water hydraulics, and underwater tools system. 